\title{
MUSIC THERAPY EXPLAINED BY THE PRINCIPLES OF NEUROPLASTICITY
}

Fulvia Anca Constantin1

\author{
Transilvania University, Braşov, 500036, Romania \\ *fulvia.constantin@gmail.com
}

\begin{abstract}
The healing influence of music was talked about and proved from long ago. Nowadays, the research done in the field of music therapy show the benefits obtained with the help of the new measuring instruments or new discoveries in neuroscience. Researchers mainly focus on quantifying changes in individual behaviors, providing evidence that music therapy is an influential intervention for a variety of clients and a variety of disorders. The aim of this paper is to explain how music therapy works, thus to find evidence regarding the neurophysiological changes associated with applying music as a therapy through the simple principles of neuroplasticity.
\end{abstract}

\section{Introduction}

In practice, music therapists usually assess the individuals' progress based upon subjective and behavioral evidence. As music therapists work across a wide range of disabilities, a full and complete understanding of the neurophysiology associated with each client's disability may be challenging. Moreover, there is a large gap of evidence regarding the neurophysiological changes associated with applying music as therapy.

\section{Defining Neuroplasticity}

According to Professor Elisabeth Stegemöller, neuroplasticity is "the ability of the brain to change throughout a person's life span as a consequence of sensory input, motor action, reward, or awareness.'[1] It occurs in the human brain on many levels, from an individual neuron, to a network of neurons or an entire region of the brain. Therefore, neuroplasticity encloses changes from individual synapses to entire cortical networks. It is known that the relative weight connections among the neurons in the network could be change (e.g. some connections are stronger than others). It is also known that an entire region of networks in the brain could be reorganized to respond to a totally new set of stimuli.

However, neuroplasticity not only refers to the emergence of new neuronal connections, but also to pruning the existent ones. It is similar to music where the relative relationship between sound and its absence (complete silence) determines the general perception and response to the music. Therefore, in neuroplasticity both, the connectivity and no connectivity of neurons, networks, and regions determine the perception and response to stimuli. The human behavior is not controlled by one single neuron, but rather by networks of neurons that often have the same function. The strength of connections between a network of neurons, as well as the level of excitability and inhibition can also change.

Information about what happens at the level of an individual neuron is provided through synaptic plasticity. Synaptic plasticity is the process by which synapses are strengthened or weakened over time. It is dependent on the level of activity existent at the synapse. A synapse is the point at which a neuronal signal is passed from one neuron to another neuron.

Neuroplasticity is not the same throughout the lifespan. There is a time in which the changes in the brain are mainly due to the formation of new connections. From birth until about the ages of 2 to 3, millions of new connections between neurons are being made. Little to no pruning is taking place. It is important to note that during this time the number of connections is increased, but not the number of neurons. It is during this time that children are "soaking" up the environment, the world around them. It is crucial for children to be exposed to appropriate 
stimuli during this time (termed "critical period" to underline its importance), as these experiences set the neuronal structure for future plasticity.

Research has shown that abnormal experience of any kind in humans can lead to abnormal patters of brain circuitry that cannot be overcome later in life. Likewise, persistent auditory deprivation and visual impairment during the critical period can lead to deficits in language or visual problems that are not overcome later in life. While the changes described in the research are extreme, it is assumed that similar mechanisms occur across other neural systems. From about the age of 3 to 6 , the brain continues to make new connections, but now there is an increase in pruning.

However, there are still substantially more new connections than pruning. Around the adolescent years, the rates between making new connections and pruning evens out and it is not until the early 20 s that the brain is completely "wired" for one's lifespan. In fact, different brain regions develop at different domes up until the early 20 s, with the motor and sensory areas being among the first regions to develop and the frontal cortex being the last area to develop. Fortunately, even though the brain is "wired" by the early 20s, neuroplasticity at all levels continues until death.

So, it is of great importance for music therapists to understand that the brain is ever changing and we possess unique instruments to create positive brain changes throughout the lifespan, from birth to death.

How Does Neuroplasticity Influence Music Therapy?

The goal of music therapy is to determine changes in a patient, either behavioral or emotional. These changes are sustained by the changes in the brain. According to professor Stegemöller there are three principles of neuroplasticity that could explain how music therapy works.

\section{Brain's Reward Circuity}

The first one is the brain's reward circuitry. Numerous studies based on research made in neurology proved that dopamine is a primary neurotransmitter involved in neuroplasticity. It has been shown to be involved in motivation and reward-seeking behavior, working memory and reinforcement learning. If before dopamine was known to be is induced by a certain food or drugs, recent neuroimaging studies showed that enjoyable music activates as well these reward networks. [1, 2] This suggests that music listening stimulates the same neural network as that involved in reinforcement learning and reward. Moreover, it had been shown a strong link between these regions and cognitive subsystems, including the orbit-frontal cortex, the area responsible for encoding the temporal aspects of memory and emotional prosodic processing.

While the results of these studies are limited to mainly listening to pleasurable music, they also demonstrate the potential of music in general when applied therapeutically to facilitate neuroplasticity.

\section{The Hebbian Principle}

The second principle in neuroplasticity is the Hebbian theory summarized by the neuroscientist $\mathrm{S}$. Löwel in two sentences: "neurons that fire together, wire together." [4] It means that, in order to make a new connection or strengthen an existing one, two neurons have to start action potentials synchronously. It was showed in numerous studies that rhythm links human behaviors to external different stimuli inducing synchrony in the neural networks supporting the behaviors. Activities such as dancing, singing, or simple deep breathing exercises make changes in the heart rate. In other words, the automatic nervous system of the brain is responsible for the regulatory processes that sustain human life, being dependent upon a certain rhythm such as sleep, heart rate and breathing.

Rhythm is an inherent property of music that often leads to entrainment, defined as "two oscillating agents that adapt a common phase and period. [5] Research has demonstrated that movement, vocalization, breathing, and heart rate can be entrained to music. However, maybe more importantly to music therapy is that, neural populations can also be entrained by sensory stimulation. While most literature regarding entrainment and brain plasticity suggests that entrainment produces an effect on 
resources regarding attention, the underlying neural mechanism of this effect remains underexplored.

The general opinion of specialists is that the Hebbian principle may indeed provide a reasonable theory for neuroplasticity with music therapy. As stated above, music therapists pair non-musical tasks, behaviors with music. Often the non-musical function is paired with rhythm. Thus, if a non-musical function is entrained with music, then it may be deduced that music is synchronizing neural populations involved in not only the areas of the brain involved in perceiving the music, but also in those areas involved in the control of the non-musical behavior. The role of a music therapist should be to pay attention to increasing "the simultaneous firing of neurons in brain areas involved in the control of those behaviors, strengthening neuronal connectivity and leading to faster and more-permanent changes in their patients." [6]

\section{Clarity of Sounds}

The third principle says that the structure of music is based on acoustic elements, so it could explain the differences in the impact of noise versus music on neuroplasticity. Music is considered to be the opposite of noise. It is calmness and harmony versus disturbance.

Music is seen as a large amount of sounds arranged more or less harmonic, the sounds are continuous and regular vibrations. What heals in music therapy is the resonance given by these vibrations. Noise is seen as being of a certain frequency spectrum (the energy or power per frequency interval.)

Researchers found the evidence of the effect given by the noise. They briefly describe the difference between the pink and the white noise as follows. The pink like noise (of $1 / \mathrm{f}$ frequency) is the frequency that carries an equal amount of noise energy. It is considered to be the most relaxing frequency, occurring widely in nature (e.g. the sound of the sea). On the other hand, the white noise is created by a range of frequencies uniformly distributed, used to increase sensitivity to regular surrounding sounds or to cover background noises. [7] Recent studies have shown that noise can negatively affect neu- roplasticity. Exposure to noise can increase stress, which is mediated by the limbic system, the area of the brain that controls emotion. As follows, the increase in stress affects both cognition and memory. Moreover, chronic exposure to noise during development can have severe implications. Changes in the auditory system, hippocampus and limbic system after exposure to noise prenatally may lead to decreased memory function and anxiety in adulthood in animal models. In contrast, exposure to music has revealed opposing affects. As proved in music therapy, long term exposure to music leads to improvements in spatial learning and enhanced learning performance.[8] This would suggest that exposure to music promotes neuroplasticity while noise suppresses neuroplasticity.

Yet, what type of music signal is best for a developing brain? This is a question hard to be answered to. Extending the results of the studies about music complexity and its impact on music therapy, we say that less complex music stimuli may be better than more complex music stimuli given the fact that more complex music stimuli carry on more "noise" within its acoustic structure.

Reasoning for why music promotes neuroplasticity in the human brain may lie in the acoustic structure of music itself. Research has suggested that the acoustic signal of a song is more consonant than that of a speech and that professional musicians have less "noise" in both their spoken and sung signals. Music therapists, trained as musicians as well, may be able to minimize the amount of noise in their vocal signal by optimizing the resonating precision in the vocal and/or instrumental sound. It is suggested that music therapists possess an unique ability to promote neuroplasticity when working with different clients just through their vocal and/or instrumental sound alone. Also, the sung text may be more easily processed in the brain than spoken text.

In order to use clear acoustic signals, to minimize the amount of noise and optimize the resonating precision of their musical sounds, it is recommended to have music therapists professionally educated like musicians playing rather well different instruments as well as to be proficient in vocal training. 


\section{Applying the Neuroplasticity Model}

Nonetheless, research has suggested that extensive music training and experience leads to changes in the brain. Thus, neuroplasticity in the brain of the clients may be provided. So is the potentially decreasing inhibition in some neurons and increasing excitation of other neurons within the neural network.

Again, pairing preferred music with emotional expression through music composition provides a synchronized dopaminergic reward that may strengthen the connections associated with related emotional expression while suppressing connectivity associated with the unrelated one. Moreover, the goals in music therapy precisely to increase social interaction, emotional expression, cognitive learning of skills, speech and communication improvement, and, not the last, to facilitate movement, are fulfilled through neuroplasticity. [9] Actually, much of what is taught and inherently known in music therapy has roots in neuroplasticity.

\section{Conclusion}

In conclusion, music is a tool that enhances neuroplasticity in the brain. Through music therapy nonmusical behavior could change, the processes governing the change in behavior leading to changes in the brain.

The potential of music therapy to change neural connectivity being explained by the three principles of neuroplasticity: the increase in dopamine, neural synchrony and a clear signal, underlie the positive effects of music therapy. This fact is once more proving the use of music by therapists to really help people with different physical and emotional disorders. Like the sculptor who meticulously adds and cuts away the material, music therapists are the artists that use music to prune and create new neural connections resulting in a beautifully crafted masterpiece revealed to the world.

\section{References}

[1] R.J.Zatorre, Musical Pleasure and Reward: Mechanisms and Dysfunctions. Annual New York Academy of Sciences. (2015) 1337. 202
[2] S. Koelsch, Brain Correlates of Music-evoked Emotions. Nature Review: Neurosciences. (2014) 15.170

[3] E. Stegemöller, Exploring the Mechanisms of Music Therapy. The Scientist. (2017) 48611

[4] E. Stegemöller, Exploring a Neuroplasticity Model of Music Therapy. Journal of music therapy. (2014) 51.211

[5] D. Levitin, This is my Brain on Music. The Science of a Human Obsession. Penguin. (2007)

[6] E. Stegemöller, Neuroplasticity May Explain the Healing Powers of Music. The Scientist, (2017) 344140

[7] D.Ursuţiu et al., Investigation of Music and Colours Influences on the Levels of Emotion and Concentration, NY REV Conference. (2016)

[8] W. Kraus et al., Music Enrichment Programs Improve the Neural Encoding of Speech in At-risk Children. Journal of Neuroscience. (2014) 34. 11913

[9] N. Salimpoor et al., Anatomically Distinct Dopamine Release During Anticipation and Experience of Peak Emotion to Music. Nature Neuroscience. (2011) 14257 